Research Article

\title{
Controllability Analysis of an Aggregate Demand Response System
}

\author{
Kazuhiro Sato and Shun-Ichi Azuma \\ Department of Systems Science, Graduate School of Informatics, Kyoto University, Kyoto 606-8501, Japan \\ Correspondence should be addressed to Kazuhiro Sato; kazuhirosato@i.kyoto-u.ac.jp
}

Received 20 February 2015; Accepted 23 June 2015

Academic Editor: Gen Qi Xu

Copyright ( $) 2015$ K. Sato and S.-I. Azuma. This is an open access article distributed under the Creative Commons Attribution License, which permits unrestricted use, distribution, and reproduction in any medium, provided the original work is properly cited.

In order to characterize the controllability of the aggregate demand response, that is, the aggregated consumer behaviors, the paper introduces the concept of controllability index which expresses the lowest occurrence probability in total electric consumption which can be changed by electric price. As the number of consumers is larger, it becomes difficult to directly analyze the controllability index. To resolve the difficulty of the large number of consumers, by using the central limit theorem, the paper approximates the controllability index and gives the solution maximizing the approximated controllability index.

\section{Introduction}

Real-time pricing (RTP) is a price based program on demand response [1-7] defined as the changes in electricity usage patterns of consumers in response to changes in electric price or to incentive payments [1]. By controlling electricity usage, consumers can produce the effectiveness in the same way that an electric power plant generates electricity. Thus it is important to implement efficacious RTP. From the viewpoint of control theory, RTP system can be regarded as a feedback system as shown in Figure 1. Here, output and reference signals are total electric consumption and electricity supply in a community, respectively, and an input signal is electric price. RTP researches can be categorized into

(i) the stability analysis of power grids under RTP $[6,8]$,

(ii) the design of electric price [9-12],

(iii) the controllability analysis of the aggregate consumers.

To clarify electric usage patterns of consumers when we can reduce the peak of total electric consumption by RTP, this paper studies (iii) which is a new problem on RTP. To this end, this paper considers that

(i) every consumer probabilistically has a value 1 or 0 , which means that a consumer uses electricity or not, (ii) probabilities of electric usages of consumers depend on electric price.

As a result, the total electric consumption which expresses the aggregate demand response also probabilistically varies corresponding to electric price.

In order to discuss the controllability of the aggregate demand response, we introduce the concept of controllability index which expresses "the lowest occurrence probability in total electric consumption which can be changed by electric price." When the controllability index is large, we can largely change the aggregate demand response by adjusting electric price.

Unfortunately, as the number of consumers is larger, the controllability index becomes complicated, and thus it is difficult to study the probabilities maximizing the controllability index. To resolve the problem, by using the central limit theorem, this paper approximates the controllability index and gives the probabilities maximizing the approximated controllability index.

Finally, we note that the recent works of $[13,14]$ are strongly related to this study. Reference [13] has studied good consumers for performing RTP by focusing on the aggregate demand response through some simulations. Reference [14] has given the design principle of randomized automated demand response machines in the viewpoint of control 


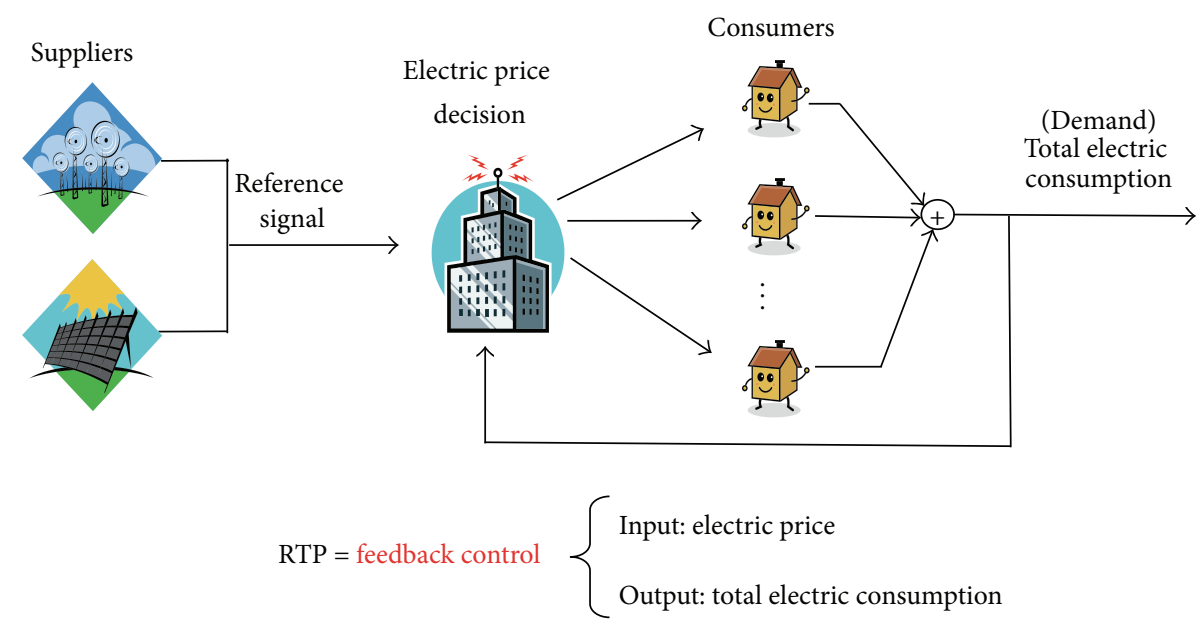

FIGURE 1: Real-time pricing system.

theory. However, $[13,14]$ have not given the performance index of the controllability of the aggregate demand response and have not mathematically studied the controllability.

The paper is organized as follows. Section 2 defines the aggregate demand response system and the controllability index. Moreover, the controllability problem is presented. Section 3 approximates the controllability index by applying the central limit theorem. Section 4 gives an approximate solution of the controllability problem. Section 5 gives a validation of the approximations used in Section 3. The conclusion is presented in Section 6.

The notation used throughout the paper is stated in Notation section.

\section{Controllability of Aggregate Demand Response}

2.1. Aggregate Demand Response System. We consider the aggregate demand response system composed of $N$ consumers as shown in Figure 2 and study its controllability to answer how total electric consumption changes by adjusting electric price.

The system corresponds to the group of consumers of the RTP system illustrated in Figure 1. For each consumer $i \in\{1,2, \ldots, N\}$, the input is electric price $u(t) \in[0, \infty)$ and the output is electric consumption $x_{i}(t) \in\{0,1\}$, where $t \in \mathbf{R}$. Here, $x_{i}(t)=0$ means that consumer $i$ does not use electricity at time $t$. On the other hand, $x_{i}(t)=1$ means that consumer $i$ uses electricity at time $t$. Then the total electric consumption is denoted by

$$
y(t):=x_{1}(t)+x_{2}(t)+\cdots+x_{N}(t) .
$$

We consider that the output $x_{i}(t)$ of consumer $i \in$ $\{1,2, \ldots, N\}$ is a random variable which has the following probability distribution:

$$
\begin{aligned}
& P\left(x_{i}(t)=1 \mid 0 \leq u(t)<\gamma\right)=p_{i}, \\
& P\left(x_{i}(t)=0 \mid 0 \leq u(t)<\gamma\right)=1-p_{i},
\end{aligned}
$$

$$
\begin{aligned}
& P\left(x_{i}(t)=1 \mid u(t) \geq \gamma\right)=q_{i}, \\
& P\left(x_{i}(t)=0 \mid u(t) \geq \gamma\right)=1-q_{i},
\end{aligned}
$$

where $p_{i}, q_{i} \in[0,1]$ are constants and $\gamma>0$. Here, $\gamma$ denotes the threshold on the electric price $u(t)$. As a result, the total electric consumption $y(t)$ also becomes a random variable which has a probability distribution.

Throughout the paper, we assume

$$
\underline{q} \leq q_{i}<p_{i} \leq \bar{p}
$$

for any $i \in\{1,2, \ldots, N\}$, where $q$ and $\bar{p}$ are constants satisfying $0<q<1,0<\bar{p}<1$, and $q<\bar{p}$. This assumption expresses the consumer buying behavior. Furthermore, we assume that the random variables $x_{1}(t), x_{2}(t), \ldots, x_{N}(t)$ are conditionally independent given $u^{*} \in[0, \infty)$; that is,

$$
\begin{gathered}
P\left(x_{1}(t)=\xi_{1}, x_{2}(t)=\xi_{2}, \ldots, x_{N}(t)=\xi_{N} \mid u(t)=u^{*}\right) \\
\quad=\prod_{i=1}^{N} P\left(x_{i}(t)=\xi_{i} \mid u(t)=u^{*}\right)
\end{gathered}
$$

for any $\xi_{1}, \xi_{2}, \ldots, \xi_{N} \in\{0,1\}$. This means that each electric usage pattern of consumers is not influenced from another consumer. The assumption is valid for the following reasons:

(i) For the aggregate demand response system illustrated in Figure 2, if consumers can communicate with each other, the independence is not satisfied. However, under the situation in which consumers cannot communicate with each other, the assumption is valid.

(ii) In the real society, the independence may not be satisfied due to the environmental factors such as the temperature and the weather. However, under a constant environment, the assumption is valid.

Remark 1 . We consider that the output $x_{i}$ of each consumer only takes two values in $\{0,1\}$. The simple setting can be 


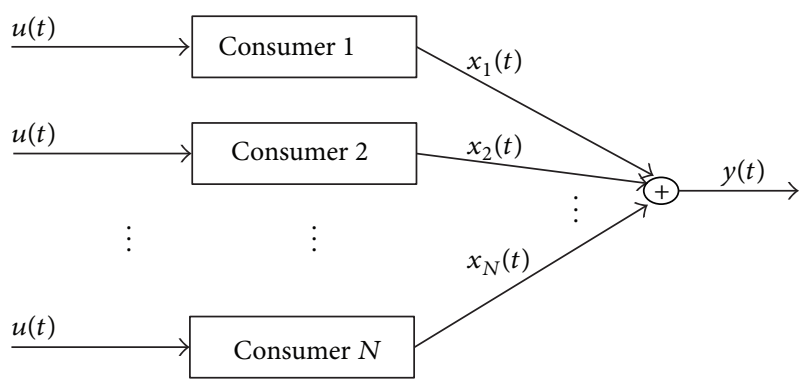

FIGURE 2: Aggregate demand response system.

considered as an approximation of the real society when we regard 1 as the average electric consumption of electric devices.

Remark 2. In this paper, we assume that electric usage of each consumer is modeled by (2). The model (2) can express the behavior of the randomized automated demand response (ADR) machine proposed in [14]. If such ADR machines prevail in households, it is meaningful to consider the model (2).

2.2. Controllability Index. In order to study the optimal consumer behavior, this paper studies the controllability of the aggregate demand response system as shown in Figure 2. As an index of controllability, we introduce

$$
C(p, q):=\min _{y^{*} \in\{0,1, \ldots, N\}} \max _{u^{*} \in[0, \infty)} c\left(p, q, y^{*}, u^{*}\right)
$$

for

$$
c\left(p, q, y^{*}, u^{*}\right):=P\left(y(t)=y^{*} \mid u(t)=u^{*}\right),
$$

where $p:=\left(p_{1}, p_{2}, \ldots, p_{N}\right) \in[q, \bar{p}]^{N}$ and $q:=\left(q_{1}, q_{2}, \ldots\right.$, $\left.q_{N}\right) \in[\underline{q}, \bar{p}]^{N}$ denote the vectors of probabilities.

The controllability index $C(p, q)$ in (5) means the lowest occurrence probability in the total electric consumption. For example, Figure 3 illustrates (6) when $N=10$ and

$$
\begin{aligned}
& p_{1}=p_{2}=\cdots=p_{10}=0.8, \\
& q_{1}=q_{2}=\cdots=q_{10}=0.15 .
\end{aligned}
$$

In Figure 3,

$$
\begin{aligned}
& \max _{u^{*} \in[0, \infty)} c\left(p, q, y^{*}, u^{*}\right) \\
& = \begin{cases}c\left(p, q, y^{*}, u^{*}\right) & \forall y^{*} \in\{5,6, \ldots, 10\} \text { if } 0 \leq u^{*}<\gamma, \\
c\left(p, q, y^{*}, u^{*}\right) & \forall y^{*} \in\{0,1, \ldots, 4\} \text { if } u^{*} \geq \gamma\end{cases} \\
& = \begin{cases}c\left(p, q, y^{*}, 0\right) & \text { if } y^{*} \in\{5,6, \ldots, 10\}, \\
c\left(p, q, y^{*}, \gamma\right) & \text { if } y^{*} \in\{0,1, \ldots, 4\}\end{cases}
\end{aligned}
$$

and thus

$$
\begin{aligned}
C(p, q) & =\min (c(p, q, 5,0), c(p, q, 4, \gamma)) \\
& =c(p, q, 5,0) \approx 0.0264 .
\end{aligned}
$$

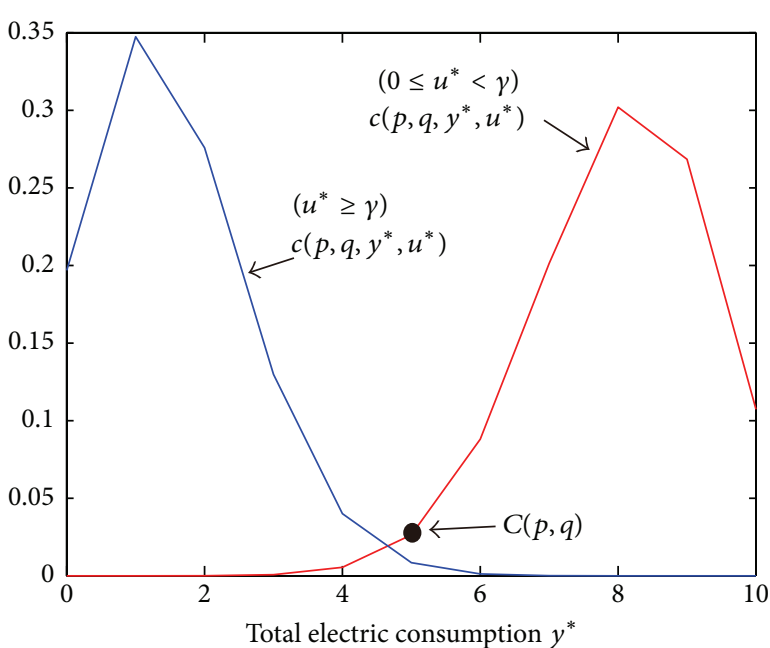

FIGURE 3: Illustration of (6) under (7).

If controllability index (5) is sufficiently large, we can approximately coincide the total electric consumption $y(t)$ with any value in $\{0,1, \ldots, N\}$ by adjusting the electric price $u(t)$. Hence if controllability index (5) is sufficiently large, we can decide to perform RTP for a community such as an apartment composed of many consumers. Furthermore, we can use the controllability index (5) as the design index of ADR machines $[14,15]$ for performing RTP. Therefore we want to consider the following problem.

Controllability Problem. Find $p, q$ maximizing the controllability index $C(p, q)$.

By solving the above problem, we can obtain the optimal consumer behavior from the viewpoint of the controllability of the aggregate demand response.

2.3. Difficulty of the Controllability Problem. As the number of consumers $N$ is larger, the optimization variables $p_{i}, q_{i}$, $i=1,2, \ldots, N$ for the controllability problem increase. As a result, when $N$ is large, we cannot solve the controllability problem even if we use any numerical methods. In fact, as shown in Appendix A, we obtain

$$
C(p, q)=\min _{y^{*} \in\{0,1, \ldots, N\}} \max \left(\widetilde{c}_{1}\left(p, y^{*}\right), \widetilde{c}_{2}\left(q, y^{*}\right)\right)
$$

for

$$
\begin{gathered}
\widetilde{c}_{1}\left(p, y^{*}\right) \\
:=\sum_{\left(\xi_{1}, \xi_{2}, \ldots, \xi_{N}\right) \in \mathbf{S}_{y^{*}}} \prod_{i=1}^{N} P\left(x_{i}(t)=\xi_{i} \mid 0 \leq u(t)<\gamma\right), \\
\widetilde{c}_{2}\left(q, y^{*}\right):=\sum_{\left(\xi_{1}, \xi_{2}, \ldots, \xi_{N}\right) \in \mathbf{S}_{y^{*}}} \prod_{i=1}^{N} P\left(x_{i}(t)=\xi_{i} \mid u(t) \geq \gamma\right),
\end{gathered}
$$


where

$$
\mathbf{S}_{y^{*}}:=\left\{\left(\xi_{1}, \xi_{2}, \ldots, \xi_{N}\right) \in\{0,1\}^{N} \mid \sum_{i=1}^{N} \xi_{i}=y^{*}\right\} .
$$

By substituting (2) into $P\left(x_{i}(t)=\xi_{i} \mid 0 \leq u(t)<\gamma\right)$ in (11) and $P\left(x_{i}(t)=\xi_{i} \mid u(t) \geq \gamma\right)$ in (12), we obtain

$$
\begin{aligned}
& \widetilde{\boldsymbol{c}}_{1}\left(p, y^{*}\right)=\sum_{\left(i_{1}, i_{2}, \ldots, i_{N}\right) \in \widetilde{\mathbf{S}}_{y^{*}}} p_{i_{1}} p_{i_{2}} \cdots p_{i_{y^{*}}}\left(1-p_{i_{y^{*}+1}}\right) \\
& \times\left(1-p_{i_{y^{*}+2}}\right) \cdots\left(1-p_{i_{N}}\right), \\
& \widetilde{\boldsymbol{c}}_{2}\left(q, y^{*}\right)=\sum_{\left(i_{1}, i_{2}, \ldots, i_{N}\right) \in \widetilde{\mathbf{S}}_{y^{*}}} q_{i_{1}} q_{i_{2}} \cdots q_{i_{y^{*}}}\left(1-q_{i_{y^{*}+1}}\right) \\
& \times\left(1-q_{i_{y^{*}+2}}\right) \cdots\left(1-q_{i_{N}}\right),
\end{aligned}
$$

where

$$
\begin{aligned}
\widetilde{\mathbf{S}}_{y^{*}} & :=\left\{\left(i_{1}, i_{2}, \ldots, i_{N}\right)\right. \\
& \in\{1,2, \ldots, N\}^{N} \mid\left(\begin{array}{cccc}
1 & 2 & \cdots & N \\
i_{1} & i_{2} & \cdots & i_{N}
\end{array}\right) \in \operatorname{Sym}(N), i_{1} \\
& \left.<i_{2}<\cdots<i_{y^{*}}, i_{y^{*}+1}<i_{y^{*}+2}<\cdots<i_{N}\right\} .
\end{aligned}
$$

Hence, the controllability index (5) is calculated by solving a minimax problem for the higher-order polynomial defined by (14) and (15).

To resolve the difficulty on the number of consumers $N$, in the next section, we approximate the controllability index (5).

\section{Approximation of the Controllability Index}

This section approximates controllability index (5) by the central limit theorem $[16,17]$ (see Appendix H).

To this end, we define

$$
\begin{aligned}
& \mu_{1}:=E(y(t) \mid 0 \leq u(t)<\gamma)=\sum_{i=1}^{N} p_{i}, \\
& \sigma_{1}:=\sqrt{V(y(t) \mid 0 \leq u(t)<\gamma)}=\sqrt{\sum_{i=1}^{N}\left(p_{i}-p_{i}^{2}\right)}, \\
& \mu_{2}:=E(y(t) \mid u(t) \geq \gamma)=\sum_{i=1}^{N} q_{i}, \\
& \sigma_{2}:=\sqrt{V(y(t) \mid u(t) \geq \gamma)}=\sqrt{\sum_{i=1}^{N}\left(q_{i}-q_{i}^{2}\right)}
\end{aligned}
$$

where $\mu_{1}$ and $\sigma_{1}$ represent the expectation and the standard deviation of the total electric consumption $y(t)$ when
$0 \leq u(t)<\gamma$ and $\mu_{2}$ and $\sigma_{2}$ represent them of $y(t)$ when $u(t) \geq \gamma$. The derivation is shown in Appendix B.

The following lemma can derived by applying the central limit theorem as shown in Appendix $\mathrm{H}$.

Lemma 3. For $i \in\{1,2\},\left(y(t)-\mu_{i}\right) / \sigma_{i}$ converges in distribution to the standard Gaussian random variable.

The proof of Lemma 3 is given in Appendix C.

Furthermore, as shown in Appendix D, we obtain

$$
\begin{aligned}
& \widetilde{c}_{1}\left(p, y^{*}\right)=P\left(y(t)=y^{*} \mid 0 \leq u(t)<\gamma\right), \\
& \widetilde{c}_{2}\left(q, y^{*}\right)=P\left(y(t)=y^{*} \mid u(t) \geq \gamma\right),
\end{aligned}
$$

where $\widetilde{c}_{1}\left(p, y^{*}\right)$ and $\widetilde{c}_{2}\left(q, y^{*}\right)$ are defined by (11) and (12), respectively.

Hence if $N$ is sufficiently large, by Lemma 3, (21), and (22), we can consider that

$$
\begin{aligned}
& \widetilde{c}_{1}\left(p, y^{*}\right) \approx f_{1}\left(y^{*}, p\right), \\
& \widetilde{c}_{2}\left(q, y^{*}\right) \approx f_{2}\left(y^{*}, q\right)
\end{aligned}
$$

for

$$
\begin{aligned}
& f_{1}\left(y^{*}, p\right):=\frac{1}{\sqrt{2 \pi} \sigma_{1}} \exp \left(-\frac{\left(y^{*}-\mu_{1}\right)^{2}}{2 \sigma_{1}^{2}}\right), \\
& f_{2}\left(y^{*}, q\right):=\frac{1}{\sqrt{2 \pi} \sigma_{2}} \exp \left(-\frac{\left(y^{*}-\mu_{2}\right)^{2}}{2 \sigma_{2}^{2}}\right) .
\end{aligned}
$$

We also write $f_{1}\left(y^{*}, p\right)$ and $f_{2}\left(y^{*}, q\right)$ as $f_{1}\left(y^{*}, p_{1}, p_{2}, \ldots, p_{N}\right)$ and $f_{2}\left(y^{*}, q_{1}, q_{2}, \ldots, q_{N}\right)$, respectively. Therefore if $N$ is sufficiently large, the controllability index (5) can be approximated as

$$
C(p, q) \approx \widetilde{C}(p, q)
$$

for

$$
\widetilde{C}(p, q):=\min _{y^{*} \in\{0,1, \ldots, N\}} \max \left(f_{1}\left(y^{*}, p\right), f_{2}\left(y^{*}, q\right)\right) .
$$

We call (26) the approximated controllability index. In Section 5, we give further validation of the approximations (23) and (25) through simulations.

\section{Controllability Analysis}

This section gives an approximate solution of the controllability problem as mentioned in Section 2.2 by using the approximated controllability index (26).

In order to study $p$ and $q$ maximizing the approximated controllability index (26), we first clarify the probabilities $p$ and $q$ such that variances of $f_{1}\left(y^{*}, p\right)$ and $f_{2}\left(y^{*}, q\right)$ are maximized under the constraints that means $f_{1}\left(y^{*}, p\right)$ and $f_{2}\left(y^{*}, q\right)$ are constants. Namely, we first solve

$$
\begin{aligned}
\max _{p_{1}, p_{2}, \ldots, p_{N}} & \sum_{i=1}^{N}\left(p_{i}-p_{i}^{2}\right) \\
\text { s.t. } & \sum_{i=1}^{N} p_{i}=\alpha N,
\end{aligned}
$$

where $0 \leq \alpha \leq 1$ 
Lemma 4. Probabilities $p_{1}, p_{2}, \ldots, p_{N}$ are the solution of the optimization problem (27) if and only if

$$
p_{i}=\alpha \quad(i=1,2, \ldots, N) .
$$

The proof of Lemma 4 is given in Appendix E.

The following lemma shows that if the mean of $f_{1}\left(y^{*}, p\right)$ is equal to that of $f_{2}\left(y^{*}, q\right)$ and if $N$ is sufficiently large, the magnitude relationship of variances between $f_{1}\left(y^{*}, p\right)$ and $f_{2}\left(y^{*}, q\right)$ corresponds to that of values of $f_{1}\left(y^{*}, p\right)$ and $f_{2}\left(y^{*}, q\right)$.

\section{Lemma 5. Let}

$$
g_{i}(y):=\frac{1}{\sqrt{2 \pi} v_{i}} \exp \left(-\frac{\left(y-m_{i}\right)^{2}}{2 v_{i}^{2}}\right), \quad i=1,2 .
$$

If $m_{1}=m_{2}=k N(0<k<1), v_{1} \geq v_{2}$, and if

$$
N>\sqrt{\frac{1}{(\beta-k)^{2}} \frac{v_{1}^{2} v_{2}^{2}}{v_{1}^{2}-v_{2}^{2}} \ln \frac{v_{1}^{2}}{v_{2}^{2}}}
$$

for $0 \leq \beta \leq 1(\beta \neq k)$, then

$$
g_{1}(\beta N) \geq g_{2}(\beta N) \text {. }
$$

The equality of (31) holds if and only if $v_{1}=v_{2}$.

The proof of Lemma 5 is given in Appendix F.

By applying Lemmas 4 and 5, we can give $p$ and $q$ maximizing the approximated controllability index (26).

Theorem 6. If $N$ is sufficiently large, $p \in(0,1)^{N}$ and $q \in$ $(0,1)^{N}$ are the solution of

$$
\max _{p, q} \widetilde{C}(p, q)
$$

if and only if

$$
\begin{aligned}
& p_{1}=p_{2}=\cdots=p_{N}=\frac{3}{4}, \\
& q_{1}=q_{2}=\cdots=q_{N}=\frac{1}{4} .
\end{aligned}
$$

The proof of Theorem 6 is given in Appendix G.

If a pair of $p$ and $q$ is composed of the solution (33), the means of $f_{1}\left(y^{*}, p\right)$ and $f_{2}\left(y^{*}, q\right)$ are $\mu_{1}=(3 / 4) N$ and $\mu_{2}=$ $(1 / 4) N$, respectively. Furthermore, then Lemma 4 implies that the variances of $f_{1}\left(y^{*}, p\right)$ and $f_{2}\left(y^{*}, q\right)$ are maximized under $\mu_{1}=(3 / 4) N$ and $\mu_{2}=(1 / 4) N$ and are $\sigma_{1}^{2}=\sigma_{2}^{2}=$ $(3 / 16) N$. As a result, the approximated controllability index $\widetilde{C}(p, q)$ in the case of $(33)$ is larger than the index $\widetilde{C}(\widehat{p}, \widehat{q})$ in

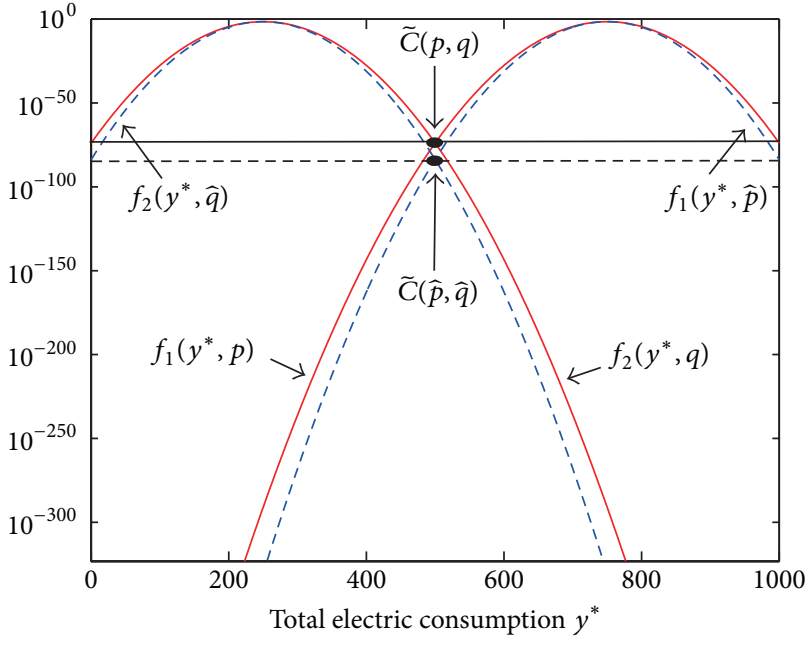

FIgure 4: Comparison with (33) and (34).

other cases with $\mu_{1}=(3 / 4) N$ and $\mu_{2}=(1 / 4) N$. For example, Figure 4 illustrates the cases of (33) and

$$
\begin{gathered}
\widehat{p}_{1}=\widehat{p}_{2}=\cdots=\widehat{p}_{500}=\frac{9}{10}, \\
\widehat{p}_{501}=\widehat{p}_{502}=\cdots=\widehat{p}_{1000}=\frac{6}{10}, \\
\widehat{q}_{1}=\widehat{q}_{2}=\cdots=\widehat{q}_{500}=\frac{1}{10}, \\
\widehat{q}_{501}=\widehat{q}_{502}=\cdots=\widehat{q}_{1000}=\frac{4}{10}
\end{gathered}
$$

when the number of consumers $N$ is 1000 . As shown in Figure 4 , the approximated controllability index $\widetilde{C}(p, q)$ in the case of (33) is larger than the index $\widetilde{C}(\hat{p}, \widehat{q})$ in the case of (34) because the variances $\sigma_{1}^{2}=\sigma_{2}^{2}=(3 / 16) N$ of $f_{1}\left(y^{*}, p\right)$ and $f_{2}\left(y^{*}, q\right)$ in the case of (33) are larger than the variances $\sigma_{1}^{2}=\sigma_{2}^{2}=(33 / 200) N$ of $f_{1}\left(y^{*}, \widehat{p}\right)$ and $f_{2}\left(y^{*}, \widehat{q}\right)$ in the case of (34). Moreover, Theorem 6 shows that if the means of $f_{1}\left(y^{*}, p\right)$ and $f_{2}\left(y^{*}, q\right)$ are not equal to $\mu_{1}=(3 / 4) N$ and $\mu_{2}=(1 / 4) N$, the approximated controllability index $\widetilde{C}(p, q)$ is smaller than the index in the case of (33). For example, Figure 5 illustrates the cases of (33) and

$$
\begin{aligned}
& \widehat{p}_{1}=\widehat{p}_{2}=\cdots=\widehat{p}_{1000}=\frac{4}{5}, \\
& \widehat{q}_{1}=\widehat{q}_{2}=\cdots=\widehat{q}_{1000}=\frac{1}{3}
\end{aligned}
$$

when the number of consumers $N$ is 1000 . As shown in Figure 5, the approximated controllability index $\widetilde{C}(p, q)$ in the case of (33) is larger than the index $\widetilde{C}(\widehat{p}, \widehat{q})$ in the case of (35).

From (25) and Theorem 6, we can give an approximate solution of the controllability problem as mentioned in Section 2.2. 


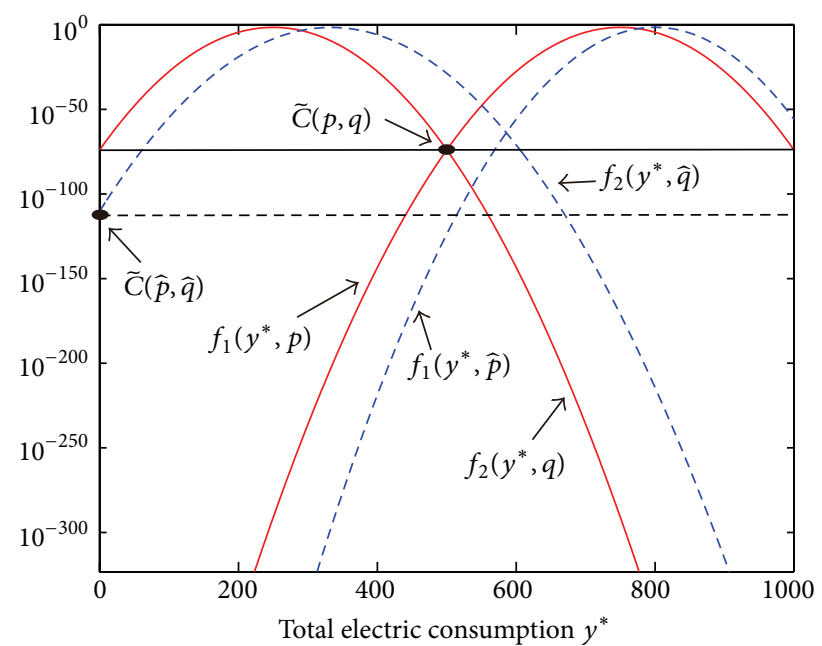

FIGURE 5: Comparison with (33) and (35).

Approximate Solution of the Controllability Problem. When the number of consumers $N$ is large, an approximate solution of the controllability problem is (33).

We note two points on the approximate solution (33):

(i) The approximate solution (33) does not depend on the number of consumers $N$; that is, we can easily implement this result into the ADR machine for performing RTP.

(ii) The approximate solution (33) does not depend on the threshold $\gamma$ on the electric price $u(t)$. This is because the controllability index $C(p, q)$ in $(5)$ is invariant for any change of the threshold $\gamma$. In fact, as shown in Section 2.3 , the controllability index $C(p, q)$ is equal to $\min _{y^{*} \in\{0,1, \ldots, N\}} \max \left(\widetilde{c}_{1}\left(p, y^{*}\right), \widetilde{c}_{2}\left(q, y^{*}\right)\right)$ in $(10)$ and the functions $\widetilde{c}_{1}\left(p, y^{*}\right)$ and $\widetilde{c}_{2}\left(q, y^{*}\right)$ are defined by (11) and (12), respectively. It seems that (11) and (12) depend on the threshold $\gamma$. However, by substituting (2) into (11) and (12), $\widetilde{c}_{1}\left(p, y^{*}\right)$ and $\widetilde{c}_{2}\left(q, y^{*}\right)$ are given in (14) and (15), which are independent of the threshold $\gamma$, respectively. Hence the controllability index $C(p, q)$ is invariant for any change of $\gamma$, and thus the approximate solution (33) does not depend on $\gamma$.

\section{Validation of the Approximations (23) and (25)}

In Section 3, the controllability index (5) has been approximated into the index (26). This section demonstrates a validation of the approximation based on simulations. To this end, first, we examine a validation of the approximation (23). Consider the maximum error of $\widetilde{c}_{1}\left(p, y^{*}\right)$ and $f_{1}\left(y^{*}, p\right)$ on $y^{*} \in\{0,1, \ldots, N\}:$

$$
\max _{y^{*} \in\{0,1, \ldots, N\}}\left|\widetilde{c}_{1}\left(p, y^{*}\right)-f_{1}\left(y^{*}, p\right)\right|
$$

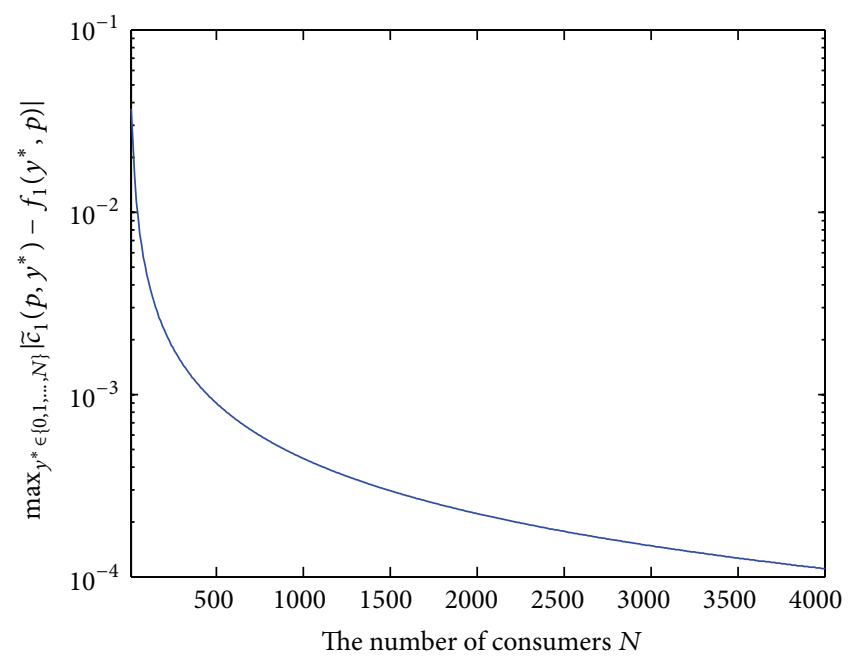

FIgURE 6: The relation of (36) and $N$ in the case of (37).

at fixed $p_{1}, p_{2}, \ldots, p_{N}$. Figures 6 and 7 illustrate the relation of (36) and $N$ in the cases of

$$
\begin{aligned}
& p_{1}=p_{2}=\cdots=p_{N}=\frac{5}{6}, \\
& p_{1}=p_{2}=\cdots=p_{N}=\frac{3}{4},
\end{aligned}
$$

respectively. On the other hand, Figures 8 and 9 illustrate the relations of (36) and $N$ in the cases of

$$
\begin{gathered}
p_{1}=p_{2}=\cdots=p_{N_{1}}=\frac{4}{5}, \\
p_{N_{1}+1}=p_{N_{1}+2}=\cdots=p_{N}=\frac{2}{3}, \\
p_{1}=p_{2}=\cdots=p_{N_{1}}=\frac{1}{2}, \\
p_{N_{1}+1}=p_{N_{1}+2}=\cdots=p_{N}=\frac{1}{3}
\end{gathered}
$$

for $N_{1}:=\operatorname{round}(N \times 0.7)$, where $\operatorname{round}(\cdot)$ denotes the round off number of $(\cdot)$. Figures $6,7,8$, and 9 show that as the number of consumers $N$ is larger, (36) becomes smaller. We have the same conclusion for different $p_{1}, p_{2}, \ldots, p_{N}$. Hence we can observe that $\widetilde{c}_{1}\left(p, y^{*}\right) \approx f_{1}\left(y^{*}, p\right)$ when $N$ is sufficiently large. Similarly, we can also observe that $\widetilde{c}_{2}\left(q, y^{*}\right) \approx f_{2}\left(y^{*}, q\right)$ when $N$ is sufficiently large. Hence if the number of consumers $N$ is sufficiently large, we can obtain the relation (23).

From the above discussion, if $N$ is sufficiently large,

$$
\begin{aligned}
& C(p, q) \\
& \quad \approx \min _{y^{*} \in\{0,1, \ldots, N\}} \max \left(f_{1}\left(y^{*}, p\right), f_{2}\left(y^{*}, q\right)\right)=\widetilde{C}(p, q)
\end{aligned}
$$

that is, we have (25). As a result, if the number of consumers $N$ is sufficiently large, the approximate solution (33) of the controllability problem is valid. 


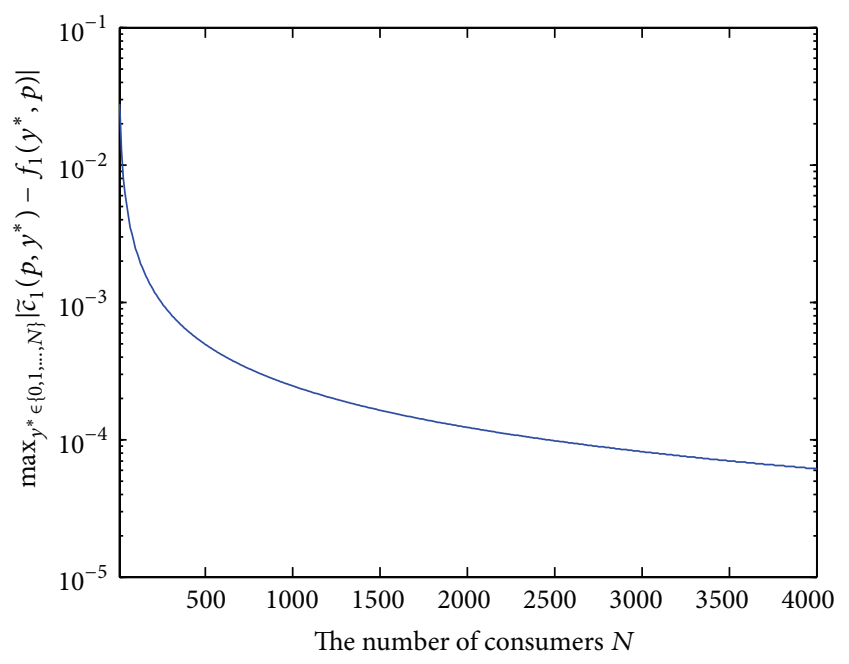

Figure 7: The relation of (36) and $N$ in the case of (38).

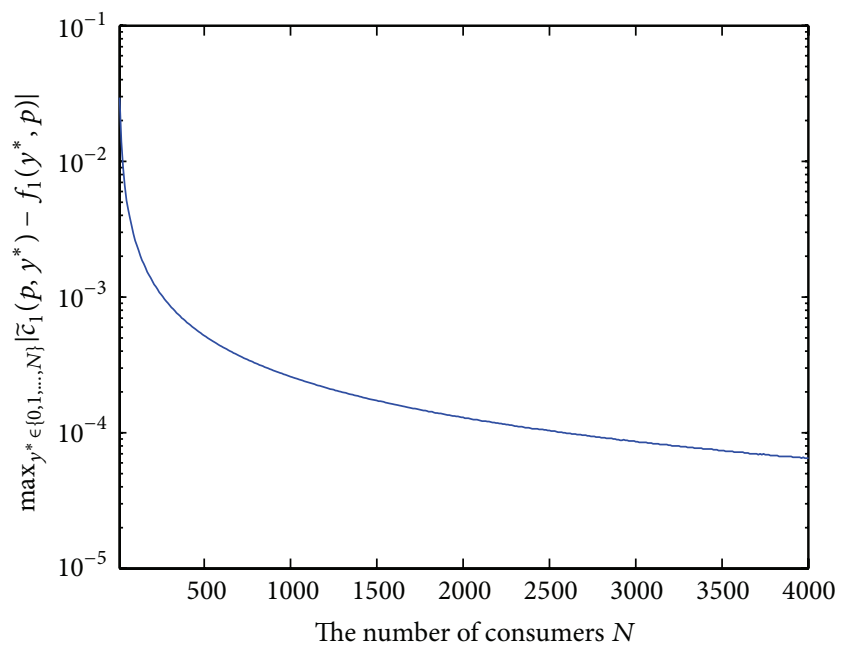

FIgURE 8: The relation of (36) and $N$ in the case of (39).

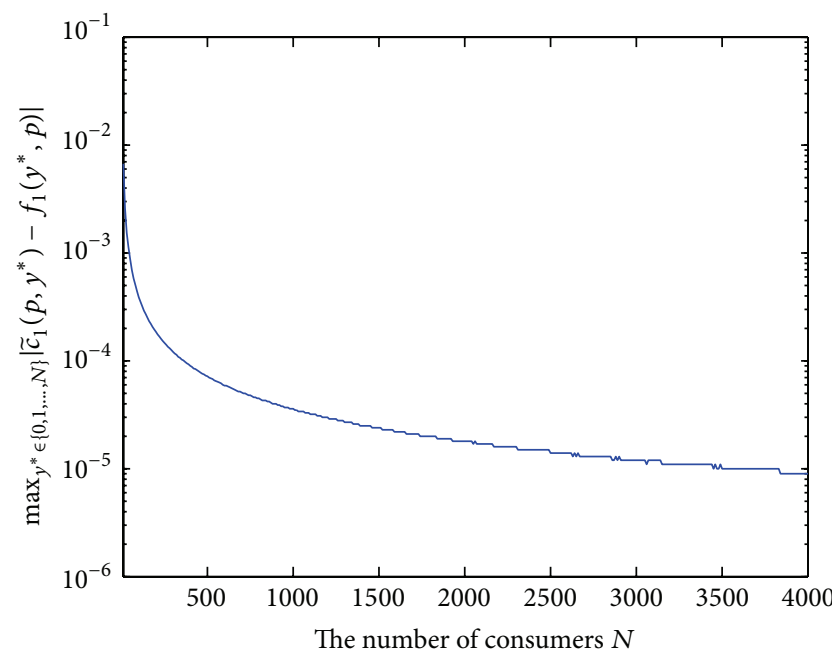

FIGURE 9: The relation of (36) and $N$ in the case of (40).

\section{Conclusion}

We have introduced the controllability index of the aggregate demand response system. By applying the central limit theorem, we have shown that if every consumer uses electricity at probability 0.75 when electric price is less than or equal to the threshold and if every consumer uses electricity at probability 0.25 when electric price is greater than the threshold, the controllability index is approximately maximized. The optimal consumer behavior can be implemented to the automated demand response machine proposed in [14] for performing RTP. Currently, we have studied the case with several thresholds on the electric price.

\section{Appendices}

\section{A. Proof of (10)}

The function $c\left(p, q, y^{*}, u^{*}\right)$ in (5) can be expressed by

$$
\begin{aligned}
& c\left(p, q, y^{*}, u^{*}\right) \\
& =P\left(\sum_{i=1}^{N} x_{i}(t)=y^{*} \mid u(t)=u^{*}\right) \\
& =\sum_{\left(\xi_{1}, \xi_{2}, \ldots, \xi_{N}\right) \in \mathbf{S}_{y^{*}}} P\left(x_{1}(t)=\xi_{1}, x_{2}(t)=\xi_{2}, \ldots, x_{N}(t)\right. \\
& \left.\quad=\xi_{N} \mid u(t)=u^{*}\right) \\
& =\sum_{\left(\xi_{1}, \xi_{2}, \ldots, \xi_{N}\right) \in \mathbf{S}_{y^{*}}} \prod_{i=1}^{N} P\left(x_{i}(\mathrm{t})=\xi_{i} \mid u(t)=u^{*}\right) .
\end{aligned}
$$

The equality (A.1) obeys (1) and (A.2) follows from the fact that each element of $S_{y^{*}}$ generates mutually exclusive events, and (A.3) obeys (4).

On the other hand, (2) implies that there exists $0 \leq u^{*}<\gamma$ such that

$$
\begin{aligned}
& P\left(x_{i}(t)=\xi_{i} \mid 0 \leq u(t)<\gamma\right) \\
& \quad=P\left(x_{i}(t)=\xi_{i} \mid u(t)=u^{*}\right)
\end{aligned}
$$

and there exists $u^{*} \geq \gamma$ such that

$$
\begin{aligned}
& P\left(x_{i}(t)=\xi_{i} \mid u(t) \geq \gamma\right) \\
& \quad=P\left(x_{i}(t)=\xi_{i} \mid u(t)=u^{*}\right) .
\end{aligned}
$$

Therefore (A.3) implies that

$$
\begin{aligned}
& \max _{0 \leq u^{*}<\gamma} c\left(p, q, y^{*}, u^{*}\right) \\
& =\max _{0 \leq u^{*}<\gamma}\left(\sum_{\left(\xi_{1}, \xi_{2}, \ldots, \xi_{N}\right) \in \mathbf{S}_{y^{*}}} \prod_{i=1}^{N} P\left(x_{i}(t)=\xi_{i} \mid u(t)=u^{*}\right)\right) \\
& =\sum_{\left(\xi_{1}, \xi_{2}, \ldots, \xi_{N}\right) \in \mathbf{S}_{y^{*}}} \prod_{i=1}^{N} P\left(x_{i}(t)=\xi_{i} \mid 0 \leq u(t)<\gamma\right) \\
& =: \widetilde{c}_{1}\left(p, y^{*}\right) .
\end{aligned}
$$


Similarly,

$$
\begin{aligned}
& \max _{u^{*} \geq \gamma} c\left(p, q, y^{*}, u^{*}\right) \\
& \quad=\sum_{\left(\xi_{1}, \xi_{2}, \ldots, \xi_{N}\right) \in \mathbf{S}_{y^{*}}} \prod_{i=1}^{N} P\left(x_{i}(t)=\xi_{i} \mid u(t) \geq \gamma\right) \\
& =: \widetilde{c}_{2}\left(q, y^{*}\right) .
\end{aligned}
$$

Equations (A.6) and (A.7) imply that

$$
\begin{aligned}
& \max _{u^{*} \in[0, \infty)} c\left(p, q, y^{*}, u^{*}\right) \\
& =\max \left(\max _{0 \leq u^{*}<\gamma} c\left(p, q, y^{*}, u^{*}\right), \max _{u^{*} \geq \gamma} c\left(p, q, y^{*}, u^{*}\right)\right) \\
& =\max \left(\widetilde{c}_{1}\left(p, y^{*}\right), \widetilde{c}_{2}\left(q, y^{*}\right)\right) .
\end{aligned}
$$

Hence (5) and (A.8) yield (10).

\section{B. Proof of (17), (18), (19), and (20)}

First, we prove (17). Equation (2) yields

$$
E\left(x_{i}(t) \mid 0 \leq u(t)<\gamma\right)=p_{i} .
$$

Hence by the definition (1) of $y(t)$,

$$
\begin{aligned}
\mu_{1} & :=E(y(t) \mid 0 \leq u(t)<\gamma) \\
& =\sum_{i=1}^{N} E\left(x_{i}(t) \mid 0 \leq u(t)<\gamma\right)=\sum_{i=1}^{N} p_{i} .
\end{aligned}
$$

Next, we prove (18). The claim that there exists $u^{*} \in[0, \gamma)$ such that $u(t)=u^{*}$ is equivalent to $0 \leq u(t)<\gamma$. Hence there exists $u^{*} \in[0, \gamma)$ such that

$$
\begin{aligned}
& V\left(x_{i}(t) \mid 0 \leq u(t)<\gamma\right)=V\left(x_{i}(t) \mid u(t)=u^{*}\right) \\
& \quad=p_{i}-p_{i}^{2} .
\end{aligned}
$$

Here, the last equality follows from (2). Therefore (4) implies that there exists $u^{*} \in[0, \gamma)$ such that

$$
\begin{aligned}
\sigma_{1} & :=\sqrt{V(y(t) \mid 0 \leq u(t)<\gamma)} \\
& =\sqrt{\sum_{i=1}^{N} V\left(x_{i}(t) \mid u(t)=u^{*}\right)}=\sqrt{\sum_{i=1}^{N}\left(p_{i}-p_{i}^{2}\right) .}
\end{aligned}
$$

Similarly, we can prove (19) and (20).

\section{Proof of Lemma 3}

Let $\bar{x}_{i}(t):=E\left(x_{i}(t) \mid 0 \leq u(t)<\gamma\right)=p_{i}$. Then (2) implies that

$$
\begin{aligned}
E & \left(\left|x_{i}(t)-\bar{x}_{i}(t)\right|^{3} \mid 0 \leq u(t)<\gamma\right) \\
& =p_{i}\left|1-p_{i}\right|^{3}+\left(1-p_{i}\right)\left|0-p_{i}\right|^{3} \\
& =\left(p_{i}-p_{i}^{2}\right)\left(1+2 p_{i}\left(p_{i}-1\right)\right) \leq\left(p_{i}-p_{i}^{2}\right) .
\end{aligned}
$$

Hence we obtain

$$
\sum_{i=1}^{N} E\left(\left|x_{i}(t)-\bar{x}_{i}(t)\right|^{3} \mid 0 \leq u(t)<\gamma\right) \leq \sigma_{1}^{2} .
$$

Therefore if

$$
\lim _{N \rightarrow \infty} \sigma_{1}=\infty
$$

then

$$
\lim _{N \rightarrow \infty} \frac{1}{\sigma_{1}^{3}} \sum_{i=1}^{N} E\left(\left|x_{i}(t)-\bar{x}_{i}(t)\right|^{3} \mid 0 \leq u(t)<\gamma\right)=0,
$$

so that the Lyapunov condition is satisfied (see Appendix $\mathrm{H}$ ). On the other hand, since we have assumed (3) and since $\sigma_{1}$ is defined by (18), (C.3) is satisfied. Hence, since the Lyapunov condition implies the Lindeberg condition, by Proposition H.1 in Appendix H, $\left(y(t)-\mu_{1}\right) / \sigma_{1}$ converges in distribution to the standard Gaussian random variable.

Similarly, by the central limit theorem, $\left(y(t)-\mu_{2}\right) / \sigma_{2}$ converges in distribution to the standard Gaussian random variable.

\section{Proof of (21) and (22)}

For all $u^{*} \in[0, \gamma)$, we have

$$
\begin{aligned}
P & \left(y(t)=y^{*} \mid u(t)=u^{*}\right) \\
& =P\left(\sum_{i=1}^{n} x_{i}(t)=y^{*} \mid u(t)=u^{*}\right) \\
& =\sum_{\left(\xi_{1}, \xi_{2}, \ldots, \xi_{N}\right) \in \mathbf{S}_{y^{*}}} \prod_{i=1}^{N} P\left(x_{i}(t)=\xi_{i} \mid u(t)=u^{*}\right) .
\end{aligned}
$$

Here, the last equality is derived from (4). Since $u^{*}$ is any value in $[0, \gamma)$ and $\widetilde{c}_{1}\left(p, y^{*}\right)$ is defined by (11), we obtain (21). Similarly, we have (22).

\section{E. Proof of Lemma 4}

Let

$$
\begin{aligned}
L\left(p_{1}, p_{2}, \ldots, p_{N}, \lambda\right):= & \sum_{i=1}^{N}\left(p_{i}-p_{i}^{2}\right) \\
& +\lambda\left(\sum_{i=1}^{N} p_{i}-\alpha N\right) .
\end{aligned}
$$

Then a necessary condition [18] for $p_{i}$ to be a solution of optimization problem (27) is

$$
\begin{aligned}
& \frac{\partial L}{\partial p_{i}}=1-2 p_{i}+\lambda=0, \quad 1 \leq i \leq N \\
& \frac{\partial L}{\partial \lambda}=\sum_{i=1}^{N} p_{i}-\alpha N=0 .
\end{aligned}
$$


Equation (E.2) implies $p_{i}=(1+\lambda) / 2$. This and (E.3) yield $\lambda=2 \alpha-1$. Thus we get $p_{i}=\alpha$.

Putting $p:=\left(p_{1}, p_{2}, \ldots, p_{N}\right)$,

$$
\frac{\partial^{2} L}{\partial p^{2}}(\alpha, \alpha, \ldots, \alpha, 2 \alpha-1)=-2 I_{N}<0
$$

where $I_{N}$ denotes the identity matrix of size $N$. Since $p_{i}=\alpha$ and $\lambda=2 \alpha-1$ satisfy (E.2) and (E.3), the relation (E.4) is a sufficient condition [18] for (28) to be a solution of optimization problem (27).

\section{F. Proof of Lemma 5}

By direct calculation,

$$
\frac{g_{1}(\beta N)}{g_{2}(\beta N)}=\frac{v_{2}}{v_{1}} \exp \left(\frac{(\beta-k)^{2} N^{2}}{2} \frac{v_{1}+v_{2}}{v_{1}^{2} v_{2}^{2}}\left(v_{1}-v_{2}\right)\right) .
$$

Thus the relation (30) is satisfied; we have (31).

\section{G. Proof of Theorem 6}

We show that if $N$ is sufficiently large, for any $p_{i}, q_{i} \in$ $(0,1)(i=1,2, \ldots, N)$,

$$
\begin{aligned}
\widetilde{C}(p, q) & \leq f_{1}\left(N, \frac{3}{4}, \frac{3}{4}, \ldots, \frac{3}{4}\right) \\
& =f_{2}\left(0, \frac{1}{4}, \frac{1}{4}, \ldots, \frac{1}{4}\right) \\
& =f_{1}\left(\frac{1}{2} N, \frac{3}{4}, \frac{3}{4}, \ldots, \frac{3}{4}\right) \\
& =f_{2}\left(\frac{1}{2} N, \frac{1}{4}, \frac{1}{4}, \ldots, \frac{1}{4}\right) \\
& =\sqrt{\frac{8}{3 \pi N}} \exp \left(-\frac{N}{6}\right)
\end{aligned}
$$

with equality if and only if (33) holds.

If $N$ is sufficiently large, for any $p_{i} \in(0,1)(i=1,2$, $\ldots, N)$ satisfying $\sum_{i=1}^{N} p_{i} \leq(3 / 4) N$,

$$
f_{1}\left(N, p_{1}, p_{2} \ldots, p_{N}\right) \leq f_{1}\left(N, \frac{3}{4}, \frac{3}{4}, \ldots, \frac{3}{4}\right) \text {. }
$$

In fact, if $N$ is sufficiently large, Lemmas 4 and 5 imply for any $p_{i} \in(0,1)(i=1,2, \ldots, N)$ satisfying $\sum_{i=1}^{N} p_{i}=\alpha N, \alpha \leq 3 / 4$,

$$
f_{1}\left(N, p_{1}, p_{2}, \ldots, p_{N}\right) \leq f_{1}(N, \alpha, \alpha, \ldots, \alpha) .
$$

Furthermore, by a direct calculation,

$$
\begin{aligned}
& \frac{f_{1}(N, 3 / 4,3 / 4, \ldots, 3 / 4)}{f_{1}(N, \alpha, \alpha, \ldots, \alpha)} \\
& \quad=\sqrt{\frac{16\left(\alpha-\alpha^{2}\right)}{3}} \exp \left(\frac{3-4 \alpha}{6 \alpha} N\right) .
\end{aligned}
$$

Hence if $N \geq(3 \alpha /(3-4 \alpha)) \ln \left(3 / 16\left(\alpha-\alpha^{2}\right)\right)$,

$$
f_{1}(N, \alpha, \alpha, \ldots, \alpha) \leq f_{1}\left(N, \frac{3}{4}, \frac{3}{4}, \ldots, \frac{3}{4}\right) .
$$

Thus if $N$ is sufficiently large, for any $p_{i} \in(0,1)(i=1,2$, $\ldots, N)$ satisfying $\sum_{i=1}^{N} p_{i} \leq(3 / 4) N,($ G.2) holds. Similarly, if $N$ is sufficiently large, for any $q_{i} \in(0,1)(i=1,2, \ldots, N)$ satisfying $\sum_{i=1}^{N} q_{i} \geq(1 / 4) N$,

$$
f_{2}\left(0, q_{1}, q_{2}, \ldots, q_{N}\right) \leq f_{2}\left(0, \frac{1}{4}, \frac{1}{4}, \ldots, \frac{1}{4}\right) .
$$

On the other hand, when $p_{i}, q_{i} \in(0,1)(i=1,2, \ldots, N)$ satisfy $\sum_{i=1}^{N} p_{i} \leq(3 / 4) N$ or $\sum_{i=1}^{N} q_{i} \geq(1 / 4) N$, (3) yields

$$
\begin{gathered}
\widetilde{C}(p, q)=\min \left(f_{1}\left(N, p_{1}, p_{2}, \ldots, p_{N}\right),\right. \\
\left.f_{2}\left(0, q_{1}, q_{2}, \ldots, q_{N}\right)\right) .
\end{gathered}
$$

Therefore if $N$ is sufficiently large, for any $p_{i}, q_{i} \in(0,1)(i=$ $1,2, \ldots, N)$ satisfying $\sum_{i=1}^{N} p_{i} \leq(3 / 4) N$ or $\sum_{i=1}^{N} q_{i} \geq(1 / 4) N$, (G.2), (G.6), and (G.7) yield

$$
\begin{aligned}
\widetilde{C} & (p, q) \\
& \leq \min \left(f_{1}\left(N, \frac{3}{4}, \frac{3}{4}, \ldots, \frac{3}{4}\right), f_{2}\left(0, \frac{1}{4}, \frac{1}{4}, \ldots, \frac{1}{4}\right)\right) \\
& =\sqrt{\frac{8}{3 \pi N}} \exp \left(-\frac{N}{6}\right) .
\end{aligned}
$$

Hence then we have (G.1).

Next, we show that if $N$ is sufficiently large, for any $p_{i}, q_{i} \in$ $(0,1)(i=1,2, \ldots, N)$ satisfying $\sum_{i=1}^{N} p_{i} \geq(3 / 4) N$ and $\sum_{i=1}^{N} q_{i} \leq(1 / 4) N,(\mathrm{G} .1)$ also holds. By Lemmas 4 and 5 , if $N$ is sufficiently large, for any $p_{i} \in(0,1)(i=1,2, \ldots, N)$ satisfying $\sum_{i=1}^{N} p_{i}=\beta N(\beta \geq 3 / 4)$,

$$
f_{1}\left(\frac{1}{2} N, p_{1}, p_{2}, \ldots, p_{N}\right) \leq f_{1}\left(\frac{1}{2} N, \beta, \beta, \ldots, \beta\right) .
$$

Moreover,

$$
\begin{aligned}
& \frac{f_{1}((1 / 2) N, \beta, \beta, \ldots, \beta)}{f_{1}((1 / 2) N, 3 / 4,3 / 4, \ldots, 3 / 4)} \\
& =\sqrt{\frac{3}{16\left(\beta-\beta^{2}\right)}} \\
& \quad \times \exp \left(-\frac{1}{2} \frac{(\beta-1 / 4)(\beta-3 / 4)}{\beta(1-\beta)} N\right) .
\end{aligned}
$$

Thus if $N \geq(\beta(1-\beta) /(\beta-1 / 4)(\beta-3 / 4)) \ln \left(3 / 16\left(\beta-\beta^{2}\right)\right)$,

$$
f_{1}\left(\frac{1}{2} N, \beta, \beta, \ldots, \beta\right) \leq f_{1}\left(\frac{1}{2} N, \frac{3}{4}, \frac{3}{4}, \ldots, \frac{3}{4}\right) .
$$

Therefore (G.9) and (G.11) imply that if $N$ is sufficiently large, for any $p_{i} \in(0,1)(i=1,2, \ldots, N)$ satisfying $\sum_{i=1}^{N} p_{i} \geq$ $(3 / 4) N$,

$$
f_{1}\left(\frac{1}{2} N, p_{1}, p_{2} \ldots, p_{N}\right) \leq f_{1}\left(\frac{1}{2} N, \frac{3}{4}, \frac{3}{4}, \ldots, \frac{3}{4}\right) .
$$


Similarly, we can show that if $N$ is sufficiently large, for any $q_{i} \in(0,1)(i=1,2, \ldots, N)$ satisfying $\sum_{i=1}^{N} q_{i} \leq(1 / 4) N$,

$$
f_{2}\left(\frac{1}{2} N, q_{1}, q_{2} \ldots, q_{N}\right) \leq f_{2}\left(\frac{1}{2} N, \frac{1}{4}, \frac{1}{4}, \ldots, \frac{1}{4}\right) .
$$

On the other hand, when $p_{i}, q_{i} \in(0,1)(i=1,2, \ldots, N)$ satisfy $\sum_{i=1}^{N} p_{i} \geq(3 / 4) N$ and $\sum_{i=1}^{N} q_{i} \leq(1 / 4) N$, (3) yields

$$
\begin{gathered}
\widetilde{C}(p, q) \leq \max \left(f_{1}\left(\frac{1}{2} N, p_{1}, p_{2}, \ldots, p_{N}\right),\right. \\
\left.f_{2}\left(\frac{1}{2} N, q_{1}, q_{2}, \ldots, q_{N}\right)\right) .
\end{gathered}
$$

Hence if $N$ is sufficiently large, for any $p_{i}, q_{i} \in(0,1)(i=$ $1,2, \ldots, N)$ satisfying $\sum_{i=1}^{N} p_{i} \geq(3 / 4) N$ and $\sum_{i=1}^{N} q_{i} \leq$ $(1 / 4) N,(G .12),(G .13)$, and (G.14) yield

$$
\begin{aligned}
\widetilde{C}(p, q) & \leq \max \left(f_{1}\left(\frac{1}{2} N, \frac{3}{4}, \frac{3}{4}, \ldots, \frac{3}{4}\right),\right. \\
\left.f_{2}\left(\frac{1}{2} N, \frac{1}{4}, \frac{1}{4}, \ldots, \frac{1}{4}\right)\right) & =\sqrt{\frac{8}{3 \pi N}} \exp \left(-\frac{N}{6}\right) .
\end{aligned}
$$

Therefore then we have (G.1).

Finally, we show that the equality condition of (G.1) is to hold (33). Lemmas 4 and 5 imply that

$$
f_{1}\left(y^{*}, p_{1}, p_{2}, \ldots, p_{N}\right)=f_{1}\left(y^{*}, \frac{3}{4}, \frac{3}{4}, \ldots, \frac{3}{4}\right)
$$

if and only if $p_{1}=p_{2}=\cdots=p_{N}=3 / 4$. Similarly, Lemmas 4 and 5 imply that

$$
f_{2}\left(y^{*}, q_{1}, q_{2}, \ldots, q_{N}\right)=f_{2}\left(y^{*}, \frac{1}{4}, \frac{1}{4}, \ldots, \frac{1}{4}\right)
$$

if and only if $q_{1}=q_{2}=\cdots=q_{N}=1 / 4$. Therefore $p_{1}, p_{2}, \ldots, p_{N}$ and $q_{1}, q_{2}, \ldots, q_{N}$ are the solution of (32) if and only if (33) holds.

\section{H. Central Limit Theorem}

For the convenience of readers, the appendix summarizes the central limit theorem. We refer [16].

A sequence of random variables $\left\{X_{i}\right\}$ is said to converge in distribution to a random variable $X$ if

$$
\lim _{n \rightarrow \infty} F_{n}(x)=F(x)
$$

for any $x \in \mathbf{R}$ at which $F$ is continuous, where $F_{n}$ and $F$ are the distribution functions of random variables $X_{n}$ and $X$, respectively. The following proposition is known as the central limit theorem [16].

Proposition H.1. Let $\left\{X_{i}\right\}$ be a sequence of independent random variables with $E\left(X_{i}\right)=\mu_{i}$ and $V\left(X_{i}\right)=\sigma_{i}^{2}$, and let

$$
\begin{aligned}
& A_{n}:=\mu_{1}+\mu_{2}+\cdots+\mu_{n}, \\
& B_{n}:=\sqrt{\sigma_{1}^{2}+\sigma_{2}^{2}+\cdots+\sigma_{n}^{2}} .
\end{aligned}
$$

If

$$
\lim _{n \rightarrow \infty} \frac{1}{B_{n}^{2}} \sum_{i=1}^{n} E\left(\left|X_{i}-\mu_{i}\right|^{2} \cdot \mathbf{1}_{\left\{\left|X_{i}-\mu_{i}\right| \geq \epsilon B_{n}\right\}}\right)=0
$$

is satisfied for any $\epsilon>0$,

$$
\frac{X_{1}+X_{2}+\cdots+X_{n}-A_{n}}{B_{n}}
$$

converges in distribution to the standard Gaussian random variable, where $\mathbf{1}_{\{.\}}$denotes the indicator function.

Note that Proposition H.1 guarantees that if condition (H.3) called the Lindeberg condition is satisfied, standardized sums of independent random variables converge in distribution to the standard Gaussian random variable without the assumption that the random variables are identically distributed.

The following condition called the Lyapunov condition is a sufficient condition for the Lindeberg condition (H.3) to hold [16]. There exists $\delta>0$ such that

$$
\lim _{n \rightarrow \infty} \frac{1}{B_{n}^{2+\delta}} \sum_{i=1}^{n} E\left(\left|X_{i}-\mu_{i}\right|^{2+\delta}\right)=0 .
$$

In fact, since $\left|X_{i}-\mu_{i}\right| \geq \epsilon B_{n}$ yields $\left|\left(X_{i}-\mu_{i}\right) / \epsilon B_{n}\right|^{\delta} \geq 1$, for any $\epsilon>0$,

$$
\begin{aligned}
& \frac{1}{B_{n}^{2}} \sum_{i=1}^{n} E\left(\left|X_{i}-\mu_{i}\right|^{2} \cdot \mathbf{1}_{\left\{\left|X_{i}-\mu_{i}\right| \geq \epsilon B_{n}\right\}}\right) \\
& \quad \leq \frac{1}{B_{n}^{2}} \sum_{i=1}^{n} E\left(\left|\frac{X_{i}-\mu_{i}}{\epsilon B_{n}}\right|^{\delta} \cdot\left|X_{i}-\mu_{i}\right|^{2} \cdot \mathbf{1}_{\left\{\left|X_{i}-\mu_{i}\right| \geq \epsilon B_{n}\right\}}\right) \\
& \quad \leq \frac{1}{\epsilon^{\delta} B_{n}^{2+\delta}} \sum_{i=1}^{n} E\left(\left|X_{i}-\mu_{i}\right|^{2+\delta}\right) .
\end{aligned}
$$

Hence if the Lyapunov condition (H.5) is satisfied, the Lindeberg condition (H.3) is also satisfied.

\section{Notation}

R: $\quad$ The set of all real numbers $\operatorname{Sym}(N)$ : The symmetric group of $N$ elements $P(A \mid B)$ : The probability of $A$ assuming $B$ $E(A \mid B)$ : The expectation of $A$ assuming $B$ $V(A \mid B)$ : The variance of $A$ assuming $B$.

\section{Conflict of Interests}

The authors declare that there is no conflict of interests regarding the publication of this paper.

\section{Acknowledgment}

This work is supported by The Japan Science and Technology Agency (JST) Strategic Basic Research Programs (CREST) 
"Creation of Fundamental Theory and Technology to Establish a Cooperative Distributed Energy Management System and Integration of Technologies across Broad Disciplines toward Social Application.”

\section{References}

[1] M. H. Albadi and E. F. El-Saadany, "A summary of demand response in electricity markets," Electric Power Systems Research, vol. 78, no. 11, pp. 1989-1996, 2008.

[2] H. A. Aalami, M. P. Moghaddam, and G. R. Yousefi, "Modeling and prioritizing demand response programs in power markets," Electric Power Systems Research, vol. 80, no. 4, pp. 426-435, 2010.

[3] A. J. Conejo, J. M. Morales, and L. Baringo, "Real-time demand response model," IEEE Transactions on Smart Grid, vol. 1, no. 3, pp. 236-242, 2010.

[4] X. Fang, S. Misra, G. Xue, and D. Yang, "Smart grid-the new and improved power grid: a survey," IEEE Communications Surveys \& Tutorials, vol. 14, no. 4, pp. 944-980, 2012.

[5] P. Faria and Z. Vale, "Demand response in electrical energy supply: an optimal real time pricing approach," Energy, vol. 36, no. 8, pp. 5374-5384, 2011.

[6] M. Roozbehani, M. A. Dahleh, and S. K. Mitter, "Volatility of power grids under real-time pricing," IEEE Transactions on Power Systems, vol. 27, no. 4, pp. 1926-1940, 2012.

[7] P. Samadi, H. Mohsenian-Rad, R. Schober, and V. W. S. Wong, "Advanced demand side management for the future smart grid using mechanism design," IEEE Transactions on Smart Grid, vol. 3, no. 3, pp. 1170-1180, 2012.

[8] D. Watts and F. L. Alvarado, "The influence of futures markets on real time price stabilization in electricity markets," in Proceedings of the 37th Annual Hawaii International Conference on System Sciences, pp. 757-764, IEEE, January 2004.

[9] A. W. Berger and F. C. Schweppe, "Real time pricing to assist in load frequency control," IEEE Transactions on Power Systems, vol. 4, no. 3, pp. 920-926, 1989.

[10] K. Kobayashi, I. Maruta, K. Sakurama, and S. Azuma, "Modeling and design of real-time pricing systems based on markov decision processes," Applied Mathematics, vol. 05, no. 10, pp. 1485-1495, 2014.

[11] A.-H. Mohsenian-Rad and A. Leon-Garcia, "Optimal residential load control with price prediction in real-time electricity pricing environments," IEEE Transactions on Smart Grid, vol. 1, no. 2, pp. 120-133, 2010.

[12] L. P. Qian, Y. J. A. Zhang, J. Huang, and Y. Wu, "Demand response management via real-time electricity price control in smart grids," IEEE Journal on Selected Areas in Communications, vol. 31, no. 7, pp. 1268-1280, 2013.

[13] K. Sato and S. Azuma, "Numerical studies of aggregate demand response by real-time pricing," in Proceedings of the 34th IASTED International Conference on Modelling, Identification and Control, Innsbruck, Austria, 2015.

[14] S. Azuma, T. Nakamoto, S. Izumi, T. Kitao, and I. Maruta, "Randomized automated demand response for real-time pricing," in Proceedings of the 6th IEEE PES Conference on Innovative Smart Grid Technologies, Washington, DC, USA, February 2015.

[15] M. A. Piette, G. Ghatikar, S. Kiliccote, P. Palensky, and C. McParland, "Open automated demand response communications specification (version 1.0)," Tech. Rep. CEC-500-2009063, California Energy Commission, PIER Program, 2009.
[16] L. Koralov and Y. G. Sinai, Theory of Probability and Random Processes, Springer, 2007.

[17] A. Papoulis and S. U. Pillai, Probability, Random Variables, and Stochastic Processes, Tata McGraw-Hill Education, 2002.

[18] R. F. Stengel, Optimal Control and Estimation, Dover, 1986. 


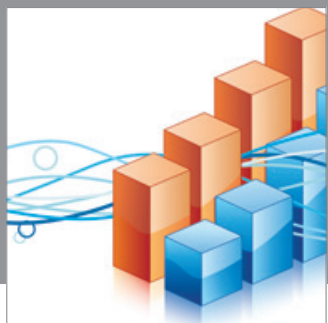

Advances in

Operations Research

mansans

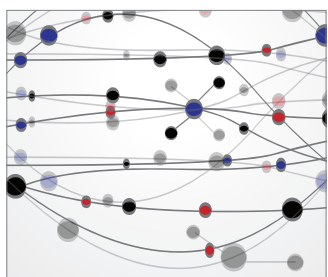

The Scientific World Journal
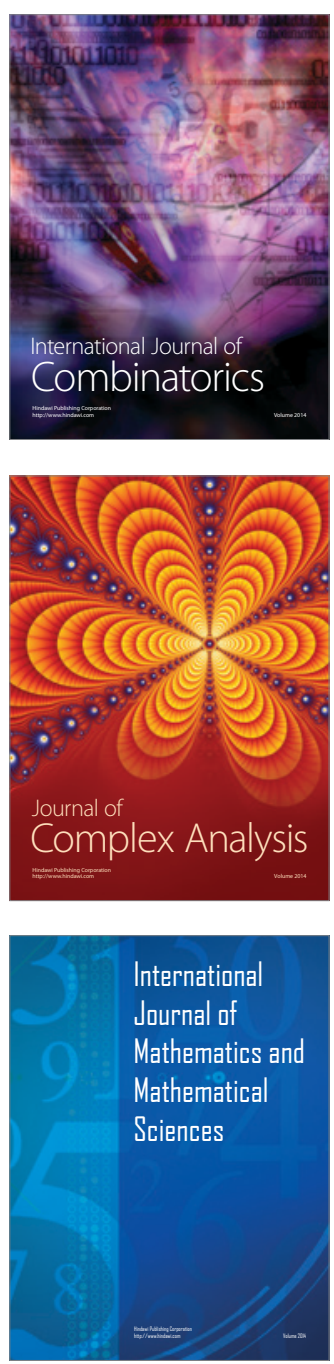
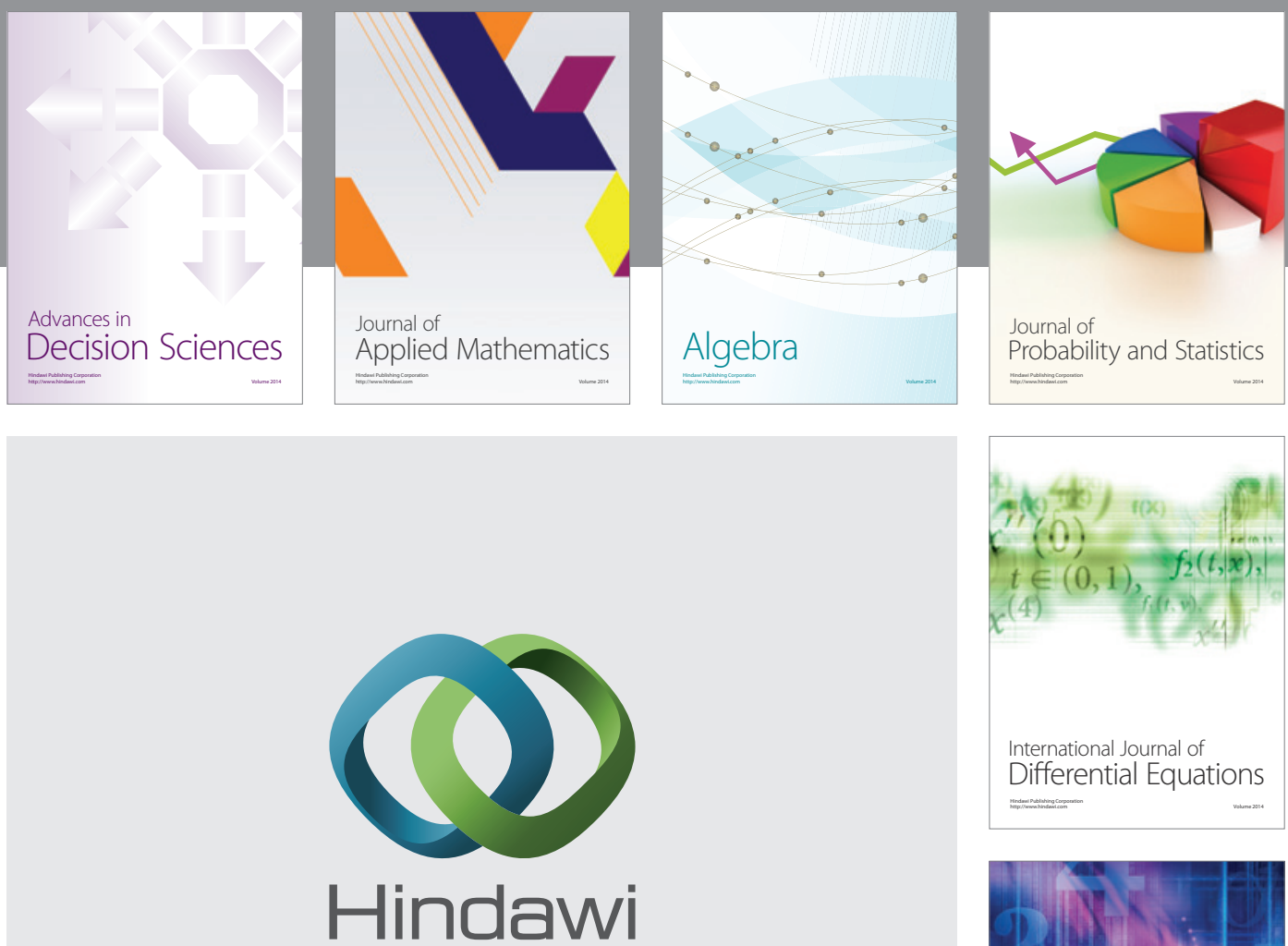

Submit your manuscripts at http://www.hindawi.com
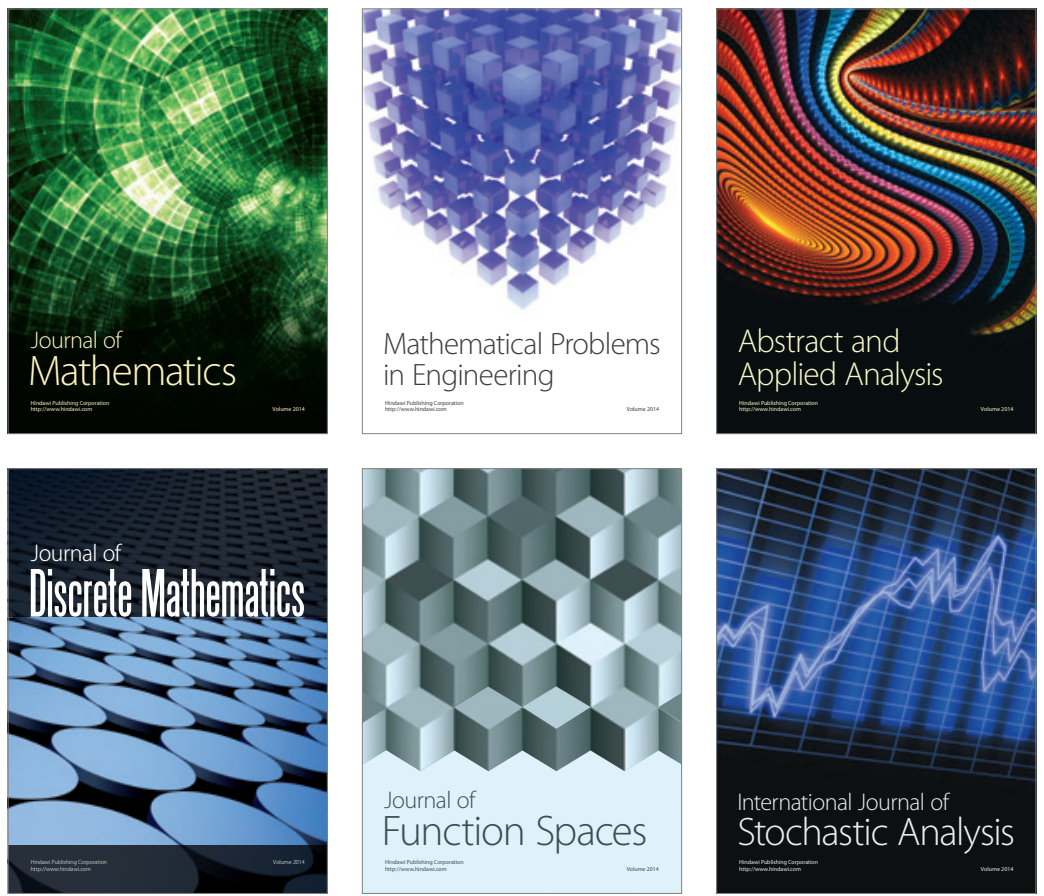

Journal of

Function Spaces

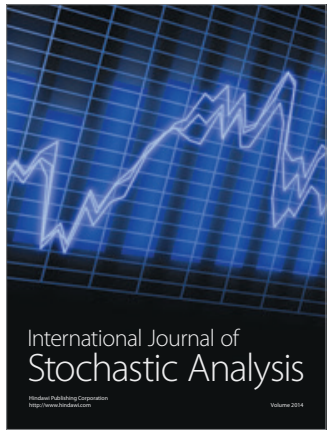

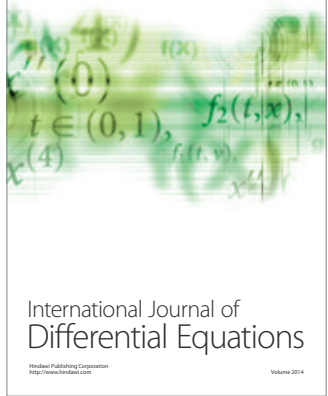
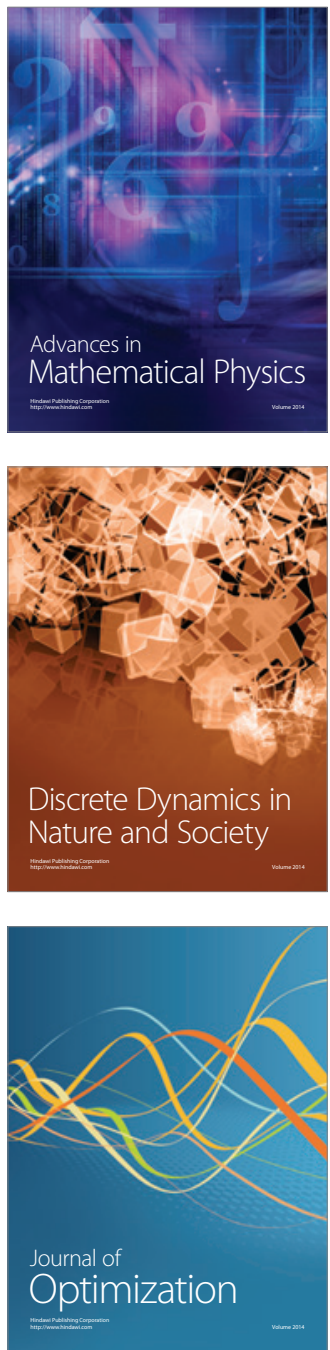